\title{
Measuring Entropy and Short-Range Correlations in the Two-Dimensional Hubbard Model
}

\author{
E. Cocchi ${ }^{1,2}$ L. A. Miller, ${ }^{1,2}$ J. H. Drewes, ${ }^{1}$ C. F. Chan, ${ }^{1}$ D. Pertot, ${ }^{1}$ F. Brennecke, ${ }^{1}$ and M. Köhl ${ }^{1}$ \\ ${ }^{1}$ Physikalisches Institut, University of Bonn, Wegelerstrasse 8, 53115 Bonn, Germany \\ ${ }^{2}$ Cavendish Laboratory, University of Cambridge, \\ JJ Thomson Avenue, Cambridge CB3 OHE, United Kingdom
}

(Received 20 February 2017; revised manuscript received 1 June 2017; published 4 August 2017)

\begin{abstract}
We measure entropy and short-range correlations of ultracold fermionic atoms in an optical lattice for a range of interaction strengths, temperatures, and fillings. In particular, we extract the mutual information between a single lattice site and the rest of the system from a comparison between the reduced density matrix of a single lattice site and the thermodynamic entropy. Moreover, we determine the single-particle density matrix between nearest neighbors from thermodynamic observables and show that even in a strongly interacting Mott insulator fermions are significantly delocalized over short distances in the lattice.
\end{abstract}

DOI: 10.1103/PhysRevX.7.031025

Quantum mechanical correlations between particles give rise to collective behavior beyond intuitive imagination. Numerous classes of many-body states whose properties occur as result of quantum correlations are known to exist, such as Bose-Einstein condensates, Mott insulators, quantum magnets, and superconductors. A general feature of a correlated many-body system on a lattice is a strong correlation between a single lattice site and its surrounding environment. These correlations induce the sensitivity and vulnerability of a many-body state to external perturbations since even a very localized perturbation can destroy the nonlocal correlations.

We explore the two-dimensional Hubbard model of spin$1 / 2$ fermionic atoms in an optical lattice. The Hubbard model considers the two elementary processes of tunneling between neighboring lattice sites with amplitude $t$ and onsite interaction between two fermions of opposite spin with strength $U$. In a single-band approximation the Hubbard Hamiltonian reads

$$
\hat{H}=-t \sum_{\langle i, j\rangle, \sigma} \hat{c}_{i \sigma}^{\dagger} \hat{c}_{j \sigma}+U \sum_{i} \hat{n}_{i \downarrow} \hat{n}_{i \uparrow} \cdot
$$

Here, $\hat{c}_{i \sigma}\left(\hat{c}_{i \sigma}^{\dagger}\right)$ denotes the annihilation (creation) operator of a fermion on lattice site $i$ in spin state $\sigma=\{\uparrow, \downarrow\}$, the bracket $\langle$,$\rangle denotes the sum over nearest neighbors, and$ $\hat{n}_{i \sigma}=\hat{c}_{i \sigma}^{\dagger} \hat{c}_{i \sigma}$ is the number operator. A Mott insulator forms at half filling and strong repulsion, i.e., for $n=\left\langle\hat{n}_{i \uparrow}\right\rangle+$ $\left\langle\hat{n}_{i \downarrow}\right\rangle=1$, and $U \gg t, k_{B} T$. It is characterized by an

Published by the American Physical Society under the terms of the Creative Commons Attribution 4.0 International license. Further distribution of this work must maintain attribution to the author(s) and the published article's title, journal citation, and DOI.
Subject Areas: Atomic and Molecular Physics,

Quantum Physics,

Quantum Information

occupation of one particle per lattice site and an energy gap for the creation of particle-hole excitations of order $U$ [1]. In contrast, for weak interactions and/or low lattice fillings, the fermions delocalize into Bloch waves and constitute a metallic state with finite charge compressibility.

Recently, antiferromagnetic correlations in the Hubbard model have been studied in several experiments [2-8]; however, access to different correlation functions sheds light on other properties of the Hubbard model, such as the charge degree of freedom. In this work, we measure both the mutual information between a single lattice site and its environment and the single-particle density matrix between nearest neighbors $\sum_{\sigma}\left\langle\hat{c}_{i, \sigma}^{\dagger} \hat{c}_{i+1, \sigma}\right\rangle$. The mutual information measures the amount of correlation between different subsystems of an optical lattice and has been measured with bosonic atoms [9]. In contrast, the single-particle density matrix is notoriously difficult to measure. In weakly interacting Bose gases, a measurement has been facilitated by interference experiments after releasing the particles from a trap [10]. However, in strongly correlated ensembles, interaction effects would severely challenge the interpretation of similar experiments. Our novel approach to measuring the single-particle density matrix is different: even though the correlations are of microscopic origin, they are macroscopically manifest in the thermodynamic observables of the system. Reversing this argument, the correlations can be determined from precise thermodynamical measurements. In the particular case of the single-particle density matrix, the corresponding thermodynamic quantity is the kinetic energy. The measurement of kinetic energy requires knowledge of both pressure and entropy, which we determine from the density profile recorded as a function of chemical potential. Previous measurements of the pressure and/or entropy in cold gases have focused on continuous (i.e., nonlattice) systems for noninteracting [11] and strongly interacting [12-17] Fermi gases. In a spin-polarized 
gas in an optical lattice, the entropy has been measured site resolved in the atomic limit, i.e., disregarding fluctuations from tunneling [18]. Our measurements extend beyond this by providing a spatially (and thus filling-)resolved detection of the entropy without the zero-tunneling approximation.

In our experiment, we prepare a spin-balanced quantum degenerate mixture of the two lowest hyperfine states $\left|F=9 / 2, m_{F}=-9 / 2\right\rangle$ and $\left|F=9 / 2, m_{F}=-7 / 2\right\rangle$ of fermionic ${ }^{40} \mathrm{~K}$ atoms $[19,20]$. We load the quantum gas into an anisotropic, three-dimensional optical lattice in which tunneling is suppressed along the vertical direction. Hence, the dynamics is restricted to two-dimensional planes within which we choose a lattice depth of $5.2(1) E_{\mathrm{rec}} \leq V_{x y} \leq$ 6.6(1) $E_{\mathrm{rec}}$, where $E_{\mathrm{rec}}=\hbar^{2} \pi^{2} /\left(2 m a^{2}\right)$ denotes the recoil energy, $a=532 \mathrm{~nm}$ is the lattice period, and $m$ is the atomic mass. The Hubbard interaction parameter $U$ is controlled by utilizing a Feshbach resonance near $202 \mathrm{G}$, which provides us with access to the parameter range from weak to strong interactions, $0 \lesssim U / t \lesssim 20$. The temperature of the gas is adjusted by heating due to a hold time in the optical lattice potential or periodic modulation of the trapping potential followed by a thermalization time. Thereby, we prepare equilibrium systems with well-defined parameters $t, U$, and $k_{B} T$. By combining radio-frequency spectroscopy and absorption imaging, we simultaneously detect the in situ density distributions of singly occupied lattice sites ("singles"), $n_{S}=\left\langle\hat{n}_{i \uparrow}-\hat{n}_{i \uparrow} \hat{n}_{i \downarrow}\right\rangle$, and doubly occupied lattice sites ("doubles"), $n_{D}=\left\langle\hat{n}_{i \uparrow} \hat{n}_{i \downarrow}\right\rangle$, in a single two-dimensional layer of the optical lattice. Our technique gives direct access to the density distribution $n(\mu)$ as a function of the chemical potential $\mu$. We perform thermometry by fitting the measured density profile $n(\mu)$ with numerical linked cluster expansion (NLCE) calculations of the two-dimensional Hubbard model [21] and the ideal $(U=0)$ Fermi gas on a square lattice.

To access the thermodynamics of the Hubbard model, we first determine the pressure from the measured density profile $n(\mu)$ [22] by employing the Gibbs-Duhem relation $S d T-A d P+N d \mu=0$, where $S$ denotes the entropy, $A$ the area, $P$ the pressure, and $N$ the total particle number. Expressing all extensive quantities in units per lattice site, pressure and density are related to each other in thermal equilibrium and at constant temperature by

$$
P(\mu, T)=\frac{1}{a^{2}} \int_{-\infty}^{\mu} n\left(\mu^{\prime}, T\right) d \mu^{\prime} .
$$

In order to limit the accumulation of technical noise in the numerical integration of the experimental data, we choose a lower bound of the integration region $\mu_{\min }$ corresponding to an average lattice site occupation of $n\left(\mu_{\min }\right)=0.01$. The resulting systematic uncertainty of the pressure is comparable to or below the statistical uncertainty of our data. In Fig. 1, we show the measured pressure as a function of the chemical potential $\mu$ [Fig. 1(a)] and as a function of $n$ [Fig. 1(b)]. We find that, for low filling, experimentally (a)

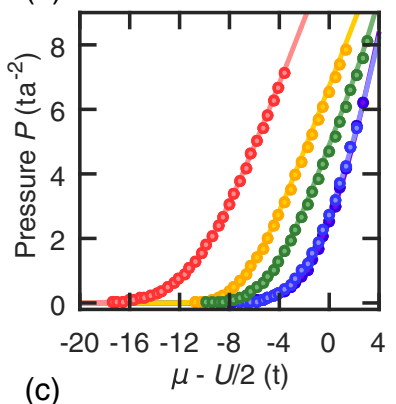

(b)
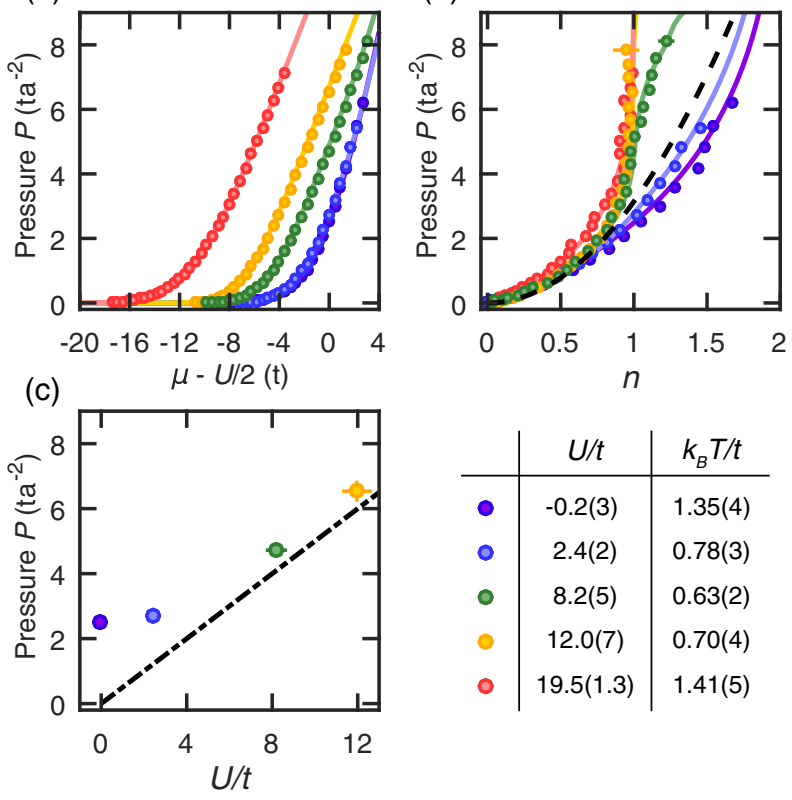

FIG. 1. Pressure as a function of interaction strength and temperature. (a) Pressure versus chemical potential for different interaction strengths and temperatures. The uncertainties of the pressure and the chemical potential are smaller or equal to the marker size. (b) Pressure versus filling for the same interactions and the same temperatures as in (a). Horizontal error bars display the standard error obtained from averaging the density data over regions of constant chemical potential. The solid lines in (a) and (b) are the predictions from NLCE data [21] with the exception of the purple solid line, which represents the ideal Fermi gas on a lattice; the black, dashed line in (b) is the $T=0$ prediction of the free Fermi gas using the effective mass at the bottom of the lowest band. (c) Pressure at half filling $n=1$ versus interaction strength. The horizontal error bars display the systematic uncertainties of $U / t$, the vertical error bars indicate the uncertainty of determining half filling from the density profiles $n(\mu)$. The dash-dotted line is the infinite- $U$ and zero-temperature prediction $P=U /\left(2 a^{2}\right)$.

determined pressures are nearly independent of interaction strength and agree well with the theoretical prediction of the free Fermi gas. We attribute this behavior to the suppression of interaction effects at low filling and the nearly harmonic dispersion relation at the bottom of the band. However, for $n \gtrsim 0.5$, we observe deviations from the free Fermi gas behavior. For weak interactions, $U / t \lesssim 3$, the pressure is smaller than that of the free Fermi gas since for $n \gtrsim 0.5$ the particles experience the nonharmonic dispersion, which affects the pressure versus density relation. For strong interactions, $U / t \gtrsim 8$, the pressure increases over that of the free Fermi gas and, in particular, develops a near-vertical slope at half filling when the lattice gas enters into a Mott insulator. This behavior is associated with the opening of the charge gap of the Mott insulator, and one can understand the pressure at half filling in the limit of zero temperature and infinite interactions by considering the internal energy $E=\langle\hat{H}\rangle=0$, which leads 
to $P=U /\left(2 a^{2}\right)$. We plot this relation in Fig. 1(c) and find asymptotic agreement with our data.

We next determine the thermodynamic entropy per site $s$ from the measured pressure at constant chemical potential:

$$
s=\left.a^{2} \frac{d P}{d T}\right|_{\mu=\text { const }} .
$$

In order to evaluate the entropy reliably, we take data sets $n(\mu)$ very finely spaced in temperature increments of $k_{B} \Delta T \sim 0.2 t$. For each data set we determine the pressure from the recorded density profile $n(\mu)$ and then perform a numerical derivative with respect to temperature at a fixed chemical potential. In order to suppress technical noise entering the numerical derivative, we interpolate the data in the temperature interval $\left[k_{B} T-t, k_{B} T+t\right]$ by a secondorder polynomial and calculate the slope of the fitted polynomial at temperature $T$. A second-order polynomial is chosen as a minimal model to account for a nonconstant heat capacity and at the same time to minimize the number of fit parameters to yield a stable fit of the data. In Fig. 2 we show a color map of the measured entropy per site as a function of both temperature and chemical potential for three different interaction strengths, $U / t=2.4,8.2,12$. For the weakest interaction shown, we do not observe a Mott insulator in the density profiles since the charge gap is washed out by the comparatively large kinetic energy at half filling. As a result, we observe the entropy per site to peak at half filling for all temperatures [Fig. 2(a)]. This is in agreement with the fact that for weak interactions the largest number of microstates is available at half filling. For strong interactions, $U / t \gtrsim 8$, and low temperatures, a Mott insulator forms at half filling, $\mu=U / 2$, surrounded by metallic phases at higher and lower chemical potential. We observe a nonmonotonic variation of entropy versus chemical potential with a local minimum at $\mu-U / 2=0$ signaling that, at constant temperature, entropy is smaller in the gapped phase and higher in the thermally connected gapless phase [Figs. 2(b) and 2(c)]. By comparison of Figs. 2(b) and 2(c), we also show that for stronger interactions, i.e., a larger gap, this effect extends to higher temperatures, as expected. We attribute the deviations between experimental and NLCE data for the lowest temperatures to the second-order polynomial fitting routine, which we confirm by analyzing NLCE data with the same routine as the experimental data and comparing to the theoretically computed entropies.

We now turn our attention to the comparison between the thermodynamic and the local entropy which quantifies the amount of correlations between a single lattice site and its environment. If one partitions a system into two subsystems $A$ and $B$, the amount of correlations between the two subsystems can be quantified by the mutual information
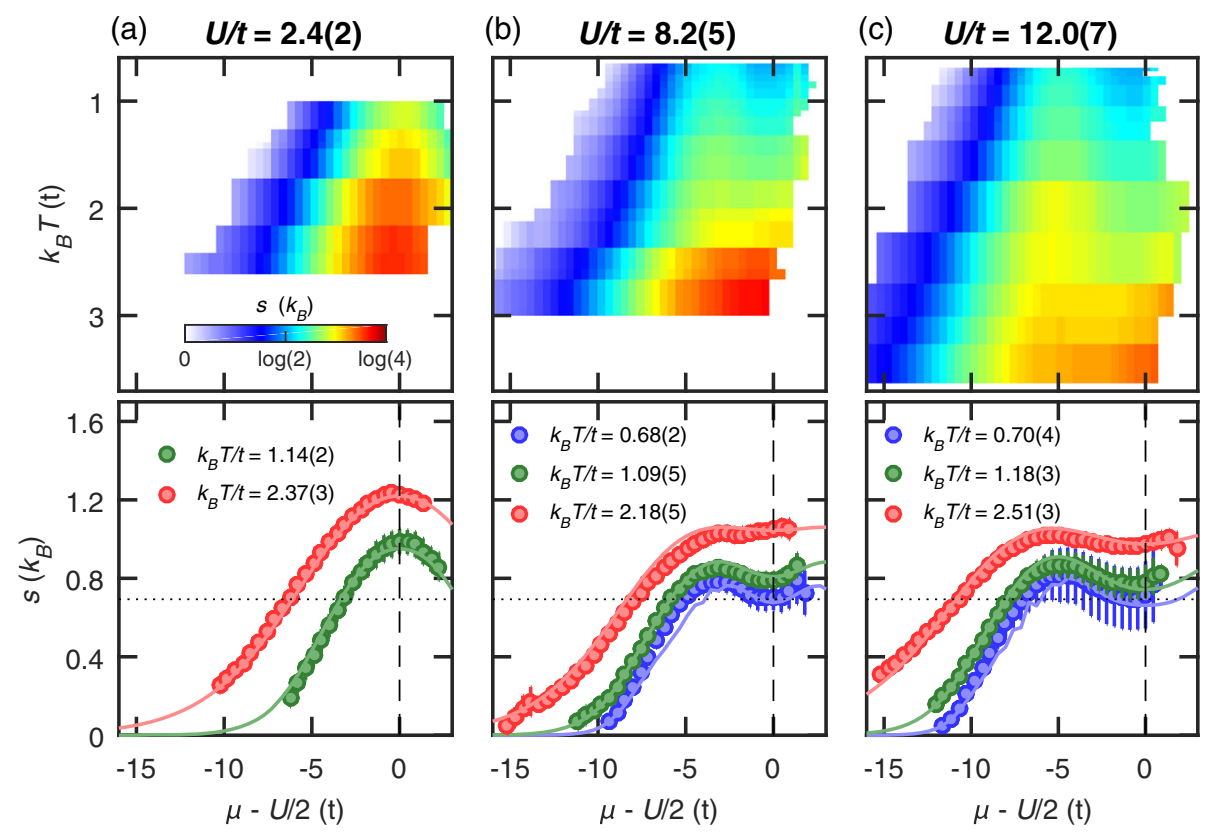

FIG. 2. Entropy per site versus chemical potential and temperature for different interaction strengths. The top row shows in color code the complete entropy data set, and the bottom row shows entropy data at selected temperatures together with the corresponding NLCE data (solid lines). The dotted horizontal line marks the value of $\log (2)$. For weak interactions (a) no Mott insulator forms and the entropy per site peaks at half filling (indicated by vertical dashed lines) at all temperatures explored. For intermediate (b) and strong (c) interactions at low temperature, one observes a local minimum of the entropy per site owing to the charge excitation gap of the Mott insulator. Horizontal errors are smaller than the marker size. The vertical error bars display the fit error of the derivative of the polynomial fit to the pressure data versus temperature. Systematic uncertainties of the entropy arising from the chosen polynomial fitting routine reach for the lowest temperatures presented values up to $0.1 k_{B}$. 
$I=S_{A}+S_{B}-S_{A B}, \quad$ where $\quad S_{X}=-k_{B} \operatorname{Tr}\left[\rho_{X} \log \left(\rho_{X}\right)\right]$ denotes the entropy of the reduced density matrix $\rho_{X}$ of subsystem $X=\{A, B\}$, and $S_{A B}$ denotes the entropy of the full system. In the following, we consider the subsystem $A$ to be a single lattice site, and subsystem $B$ to be the thermodynamic bulk excluding the single site $A$. The entropy $s_{0} \equiv s_{A}$ of a single lattice site is directly determined from the single-site reduced density matrix by $s_{0}=$ $-k_{B} \sum_{i} p_{i} \log \left(p_{i}\right)$. Here, $i=\{\uparrow \downarrow, \uparrow, \downarrow, 0\}$ labels the probabilities $p_{i}$ for a site to be occupied with either two particles, a spin-up particle, a spin-down particle, or no particles, respectively. These probabilities are directly determined from the measured singles and doubles density distributions as $p_{\uparrow \downarrow}=n_{D}, \quad p_{\uparrow}=p_{\downarrow}=n_{S}$, and $p_{0}=1-2 n_{S}-n_{D}$ [23]. The entropy of the entire system with $L \gg 1$ sites is $S_{A B}=L s$, where $s$ is the measured thermodynamic entropy per site and, likewise, $S_{B}=(L-1) s$. Hence, we obtain the mutual information as $I=s_{0}-s$, which we plot in Fig. 3 for various fillings, temperatures, and interaction strengths. For low temperatures, we find a mutual information greater than zero, which indicates correlations between the single lattice site and its environment. We observe that for low filling

(a)
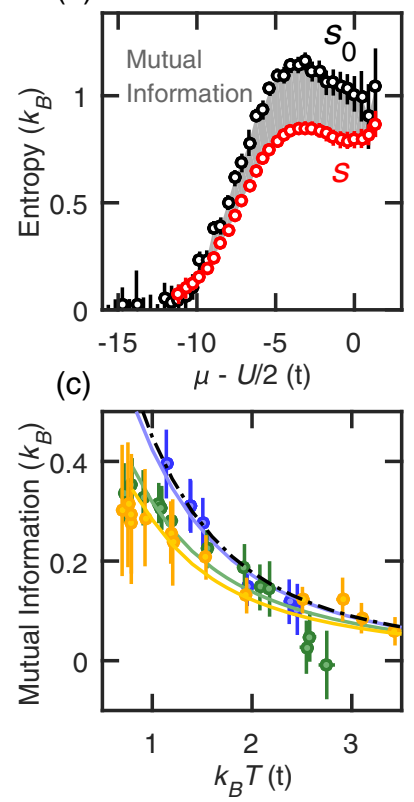

(d)
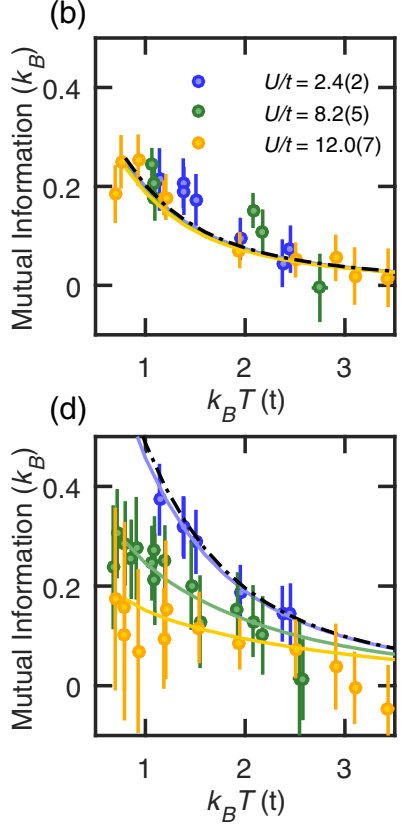

FIG. 3. Mutual information between a single lattice site and its surrounding environment for different fillings, interactions, and temperatures. (a) Data used to extract the mutual information, for the exemplary case of $U / t=8.2(5)$ and $k_{B} T / t=1.09(5)$. The mutual information is given by the difference between the singlesite entropy $s_{0}$ and the thermodynamic entropy per site $s$. Mutual information for $n=0.20$ (2) (b), $n=0.67(2)$ (c), and $n=$ $1.00(2)(d)$. The lines are theory predictions extracted from NLCE data (solid lines) and for the noninteracting Fermi gas on a lattice (dash-dotted lines). The color code is the same for all plots. Error bars display the statistical measurement uncertainty.
[Fig. 3(b)], for which the effects of interactions are generally weak, the mutual information is mostly independent of the interaction strength. In contrast, at half filling [Fig. 3(d)] we observe a larger mutual information for weak interactions than for strong interactions. For high temperatures, the mutual information approaches zero, indicating the absence of any correlations.

In order to gain further insight into the nature of the correlations between a single site and its environment, we study the first-order correlation function $G^{(1)}(1)=$ $\sum_{\sigma}\left\langle\hat{c}_{i, \sigma}^{\dagger} \hat{c}_{i+1, \sigma}\right\rangle$ between neighboring lattice sites $i$ and $i+1$. The first-order correlation function, normalized to the density $G^{(1)}(0)=n$, measures the degree of delocalization of the fermions and has not been measured in optical-lattice experiments before. In the tight-binding approximation, which is well fulfilled in our optical lattice, $G^{(1)}(1)$ is available through a measurement of the kinetic energy of site $i, E_{k, i} \equiv-t \sum_{j, \sigma}\left\langle\hat{c}_{i \sigma}^{\dagger} \hat{c}_{j \sigma}\right\rangle \delta_{|i-j|, 1}=-4 t G^{(1)}(1)$. We deduce the kinetic energy from spatially resolved measurements of the total energy of the lattice gas, combining the measurements of entropy, pressure, and density, and the interaction energy given by $U n_{D}$ :

$$
E_{k}=\left(s T+\mu n-P a^{2}\right)-U n_{D} .
$$

In Fig. 4, we plot the magnitude of the kinetic energy for the different interactions investigated. For weak interactions the magnitude of the kinetic energy peaks at half filling, similar to the mutual information (Fig. 3). Hence, we identify delocalization as the mechanism to provide the correlation between a single lattice site and the environment for the lowest interaction. Conversely, for stronger interactions the shape of the kinetic-energy curves changes qualitatively as the peak shifts to a lower filling because of the emerging Mott gap. At half filling the magnitude of the kinetic energy decreases with increasing interaction strength, signaling the crossover towards a localized, Mott-insulating state.

The measured nearest-neighbor correlations provide a first glimpse at the single-particle density matrix $G^{(1)}(|i-j|)=$ $\sum_{\sigma}\left\langle\hat{c}_{i \sigma}^{\dagger} \hat{c}_{j \sigma}\right\rangle$ in the lattice. With the measurements shown here, we can determine the single-particle density matrix for the values $|i-j|=0,1$. Concentrating on the case of half filling (even though the discussion can be generalized to any filling), we find $G^{(1)}(0)=1$, and in the case of the metallic phase $(U / t=2.4), G^{(1)}(1)=0.29(2)$. This drop is in line with the expected thermal de Broglie wavelength, which at a temperature of $k_{B} T=t$ is approximately $\lambda_{\mathrm{dB}} \simeq a$. For higher temperature, the spatial correlations drop correspondingly faster. In contrast, for a Mott insulator $(U / t=12)$, the drop is from $G^{(1)}(0)=1$ to $G^{(1)}(1)=$ $0.11(5)$ even though the temperature is even lower. This indicates localization of the fermions with increasing interaction strength. We note, however, that even for the 

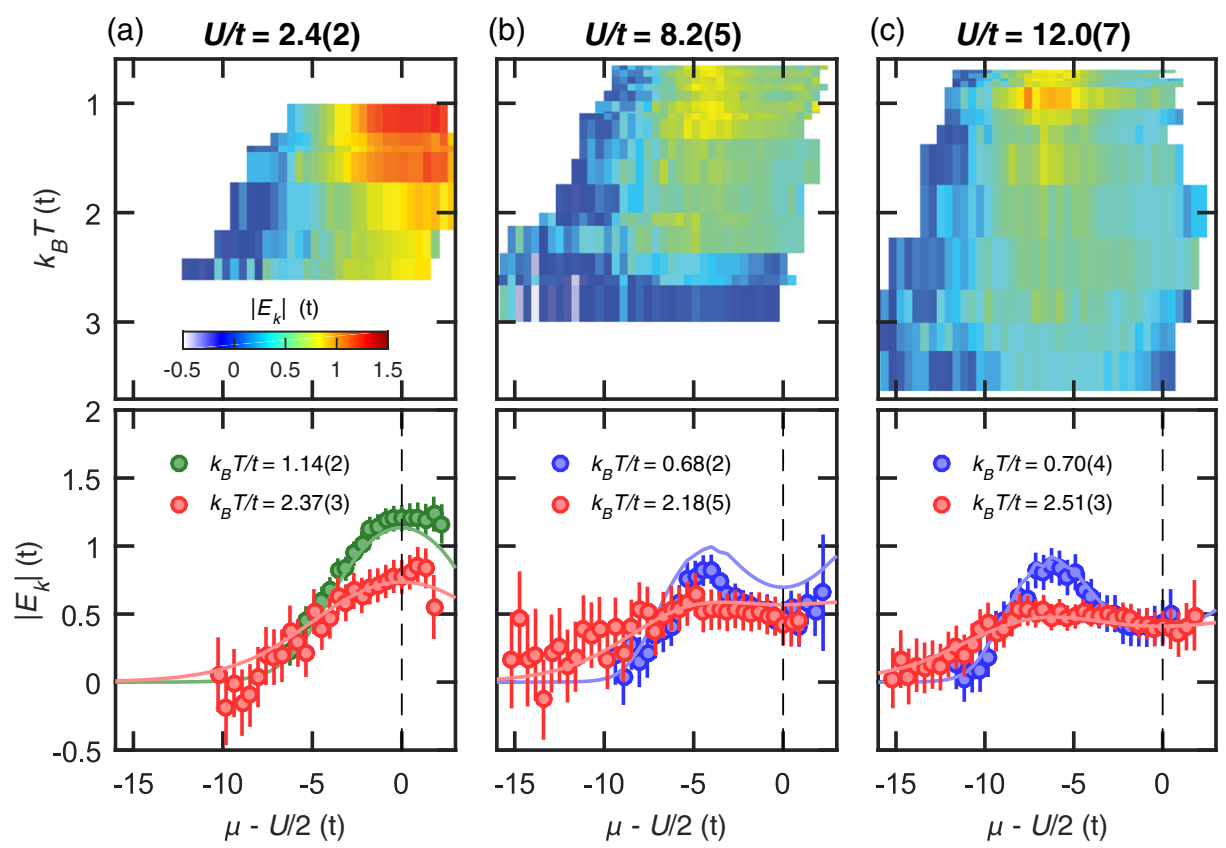

FIG. 4. Kinetic energy versus chemical potential and temperature. The top row shows in color code the complete kinetic energy data set, and the bottom row shows cuts at selected temperatures together with the corresponding NLCE theory (solid lines). While the atoms are maximally delocalized for weak interactions, for strong interactions the delocalization is only reduced, but not fully suppressed. Error bars display the statistical measurement uncertainty; see Appendix.

strongest interaction explored by our experiment, the atoms are not fully localized [24] even though the compressibility of the state is almost zero [20].

In conclusion, we measure short-range correlations in the two-dimensional Hubbard model and show that at low temperatures a single lattice site develops correlations with the surrounding environment. In particular, we find that even for strongly interacting Mott insulators with a vanishing compressibility the fermions are still significantly delocalized over neighboring lattice sites. We note that the technique we present here determines the full thermodynamic entropy, including the entropy in the spin sector without the need for spin-resolved measurements. Hence, it could find use in future attempts to cool strongly correlated quantum gases by reshuffling the entropy $[8,25,26]$.

We thank A. Daley and C. Kollath for discussions. The work has been supported by DFG (SFB/TR 185), the Alexander von Humboldt Stiftung, EPSRC, and ERC (Grant No. 616082).

\section{APPENDIX: ERROR ANALYSIS}

Here, we specify the uncertainties of the measured quantities and Hubbard parameters and provide details about the error analysis of the derived thermodynamic quantities.

The statistical uncertainties of the measured singles and doubles density $n_{S, D}$ are given by the standard error resulting from averaging the recorded images over regions of constant chemical potential with a bin size of $\Delta \mu=h \times 100 \mathrm{~Hz}$. The uncertainty of the total density $n=$ $2\left(n_{S}+n_{D}\right)$ is then obtained by adding the individual uncertainties in quadrature. The systematic uncertainties of the Hubbard parameters $U$ and $t$ are derived from the calibration uncertainties of the lattice depth along the three directions and the uncertainty in the parametrization of the Feshbach resonance [20]. Statistical uncertainties of the temperature $T$ and the chemical potential $\mu_{\mathrm{HF}}=U / 2$ at half filling are obtained from simultaneously fitting the recorded singles and doubles density profiles $n_{S, D}(\mu)$ to numerical simulations of the two-dimensional Hubbard model [21] or the ideal $(U=0)$ Fermi gas on a square lattice. The systematic error of $\mu_{\mathrm{HF}}$ is negligible as the maximum of the singles density $n_{S}(\mu)$ provides a modelindependent calibration of the half-filling point. The extracted temperatures are confirmed within the statistical uncertainties by fitting the low-filling regions of the density profile to the ideal $(U=0)$ Fermi gas on a square lattice.

The statistical uncertainty of the pressure $P(\mu)$ is obtained by adding the individual statistical uncertainties of the total density $n(\mu)$ in quadrature. The systematic uncertainty of the chemical potential axis is converted into an additional systematic uncertainty of the pressure only in Fig. 1(c), where the pressure at half filling is shown.

The statistical uncertainty of the entropy $s(\mu)$ is obtained from the fit errors of the second-order polynomial fit to the pressure data $P(T)$ for a given $\mu$. Systematic uncertainties of the entropy originating from the chosen temperature interval 

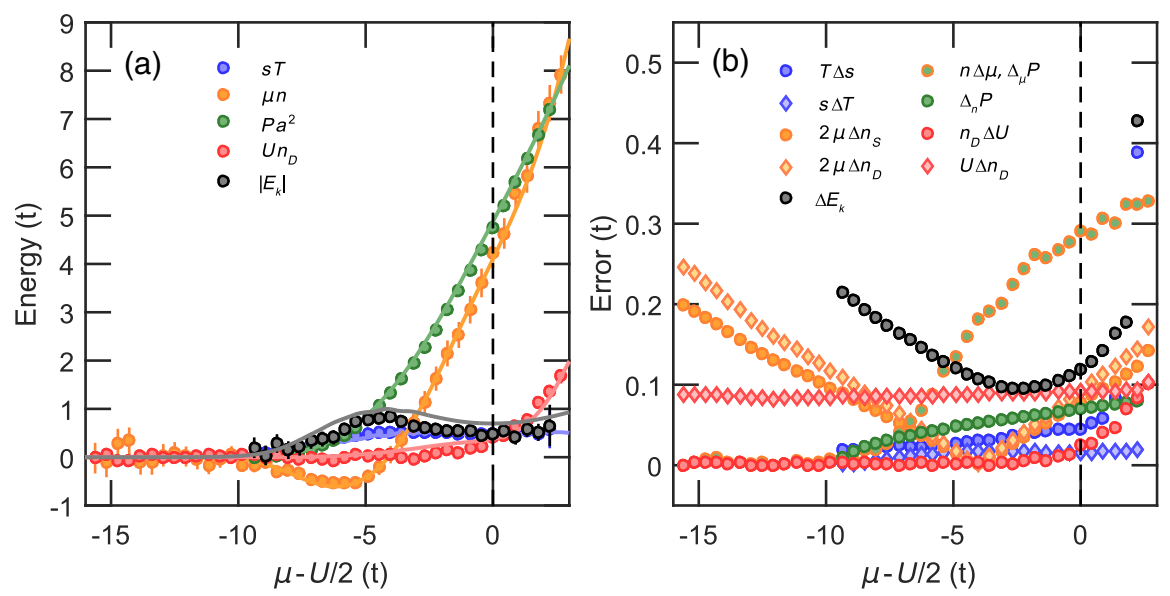

FIG. 5. Average values (a) and uncertainties (b) of the individual terms contributing to the magnitude of the kinetic energy $\left|E_{k}\right|$. Note that in the calculation of the error of the kinetic energy $\Delta E_{k}$ the term $n \Delta \mu$ cancels with the pressure uncertainty $\Delta_{\mu} P$ concerning the uncertainty of the chemical potential. The term $\Delta_{n} P$ denotes the pressure uncertainty due to the uncertainty of the density $n$. The solid lines show the theoretical expectation of the individual terms obtained from NLCE data of the two-dimensional Hubbard model [21].

and the order of the polynomial fit function have been estimated by applying the same routine to numerical density data of the two-dimensional Hubbard model [21]. In the explored temperature regime, we obtain a maximum deviation between the theoretical value of the entropy and the value obtained with our routine of $0.1 k_{B}$. The statistical uncertainty of the single-site entropy $s_{0}(\mu)$ is directly obtained from the uncertainties of the singles and doubles densities $n_{S, D}$.

In the calculation of the uncertainty of the kinetic energy [see Eq. (4)], we take into account the correlation between the terms $\mu n$ and $P a^{2}$ regarding a variation of the chemical potential. This can be seen by calculating the total differential of the kinetic energy where the two terms $n d \mu$ and $-d P a^{2}=-n d \mu$ cancel. Therefore, the uncertainty of the kinetic energy does not depend on the uncertainty of the chemical potential as long as the density $n$ does not vary significantly within the chemical potential error interval. For clarity, we plot in Fig. 5 the average values and corresponding uncertainties of the individual terms contributing to the average value and uncertainty of the kinetic energy for the representative parameter set $U / t=8.2(5)$ and $k_{B} T / t=0.68(2)$.

[1] M. Imada, A. Fujimori, and Y. Tokura, Metal-Insulator Transitions, Rev. Mod. Phys. 70, 1039 (1998).

[2] D. Greif, T. Uehlinger, G. Jotzu, L. Tarruell, and T. Esslinger, Short-Range Quantum Magnetism of Ultracold Fermions in an Optical Lattice, Science 340, 1307 (2013).

[3] R. A. Hart, P. M. Duarte, T.-L. Yang, X. Liu, T. Paiva, E. Khatami, N. Trivedi, R. T. Scalettar, D. A. Huse, and R. G. Hulet, Observation of Antiferromagnetic Correlations in the Hubbard Model with Ultracold Atoms, Nature (London) 519, 211 (2015).
[4] M. F. Parsons, A. Mazurenko, C. S. Chiu, G. Ji, D. Greif, and M. Greiner, Site-Resolved Measurement of the Spin-Correlation Function in the Fermi-Hubbard Model, Science 353, 1253 (2016).

[5] M. Boll, T. A. Hilker, G. Salomon, A. Omran, J. Nespolo, L. Pollet, I. Bloch, and C. Gross, Spin- and Density-Resolved Microscopy of Antiferromagnetic Correlations in FermiHubbard Chains, Science 353, 1257 (2016).

[6] L. W. Cheuk, M. A. Nichols, K. R. Lawrence, M. Okan, H. Zhang, E. Khatami, N. Trivedi, T. Paiva, M. Rigol, and M. W. Zwierlein, Observation of Spatial Charge and Spin Correlations in the 2D Fermi-Hubbard Model, Science 353, 1260 (2016).

[7] J. H. Drewes, L. A. Miller, E. Cocchi, C. F. Chan, N. Wurz, M. Gall, D. Pertot, F. Brennecke, and M. Köhl, Antiferromagnetic Correlations in Two-Dimensional Fermionic Mott-Insulating and Metallic Phases, Phys. Rev. Lett. 118, 170401 (2017).

[8] A. Mazurenko, C. S. Chiu, G. Ji, M. F. Parsons, M. KanászNagy, R. Schmidt, F. Grusdt, E. Demler, D. Greif, and M. Greiner, A Cold-Atom Fermi-Hubbard Antiferromagnet, Nature (London) 545, 462 (2017).

[9] R. Islam, R. Ma, P. M. Preiss, M. Eric Tai, A. Lukin, M. Rispoli, and M. Greiner, Measuring Entanglement Entropy in a Quantum Many-Body System, Nature (London) 528, 77 (2015).

[10] I. Bloch, T. W. Hänsch, and T. Esslinger, Measurement of the Spatial Coherence of a Trapped Bose Gas at the Phase Transition, Nature (London) 403, 166 (2000).

[11] A. G. Truscott, K. E. Strecker, W. I. McAlexander, G. B. Partridge, and R. G. Hulet, Observation of Fermi Pressure in a Gas of Trapped Atoms, Science 291, 2570 (2001).

[12] S. Nascimbene, N. Navon, K. J. Jiang, F. Chevy, and C. Salomon, Exploring the Thermodynamics of a Universal Fermi Gas, Nature (London) 463, 1057 (2010).

[13] M. J. H. Ku, A. T. Sommer, L. W. Cheuk, and M. W. Zwierlein, Revealing the Superfluid Lambda Transition in 
the Universal Thermodynamics of a Unitary Fermi Gas, Science 335, 563 (2012).

[14] V. Makhalov, K. Martiyanov, and A. Turlapov, GroundState Pressure of Quasi-2D Fermi and Boses Gases, Phys. Rev. Lett. 112, 045301 (2014).

[15] K. Fenech, P. Dyke, T. Peppler, M. G. Lingham, S. Hoinka, $\mathrm{H}$. $\mathrm{Hu}$, and C. J. Vale, Thermodynamics of an Attractive $2 \mathrm{D}$ Fermi Gas, Phys. Rev. Lett. 116, 045302 (2016).

[16] K. Martiyanov, T. Barmashova, V. Makhalov, and A. Turlapov, Pressure Profiles of Nonuniform Two-Dimensional Atomic Fermi Gases, Phys. Rev. A 93, 063622 (2016).

[17] L. Luo, B. Clancy, J. Joseph, J. Kinast, and J. E. Thomas, Measurement of the Entropy and Critical Temperature of a Strongly Interacting Fermi Gas, Phys. Rev. Lett. 98, 080402 (2007).

[18] A. Omran, M. Boll, T. A. Hilker, K. Kleinlein, G. Salomon, I. Bloch, and C. Gross, Microscopic Observation of Pauli Blocking in Degenerate Fermionic Lattice Gases, Phys. Rev. Lett. 115, 263001 (2015).

[19] B. Fröhlich, M. Feld, E. Vogt, M. Koschorreck, W. Zwerger, and M. Köhl, Radio-Frequency Spectroscopy of a Strongly Interacting Two-Dimensional Fermi Gas, Phys. Rev. Lett. 106, 105301 (2011).
[20] E. Cocchi, L. A. Miller, J. H. Drewes, M. Koschorreck, D. Pertot, F. Brennecke, and M. Köhl, Equation of State of the Two-Dimensional Hubbard Model, Phys. Rev. Lett. 116, 175301 (2016).

[21] E. Khatami and M. Rigol, Thermodynamics of Strongly Interacting Fermions in Two-Dimensional Optical Lattices, Phys. Rev. A 84, 053611 (2011).

[22] T.-L. Ho and Q. Zhou, Obtaining the Phase Diagram and Thermodynamic Quantities of Bulk Systems from the Densities of Trapped Gases, Nat. Phys. 6, 131 (2010).

[23] P. Zanardi, Quantum Entanglement in Fermionic Lattices, Phys. Rev. A 65, 042101 (2002).

[24] C. N. Varney, C.-R. Lee, Z. J. Bai, S. Chiesa, M. Jarrell, and R. T. Scalettar, Quantum Monte Carlo Study of the TwoDimensional Fermion Hubbard Model, Phys. Rev. B 80, 075116 (2009).

[25] J.-S. Bernier, C. Kollath, A. Georges, L. De Leo, F. Gerbier, C. Salomon, and M. Köhl, Cooling Fermionic Atoms in Optical Lattices by Shaping the Confinement, Phys. Rev. A 79, 061601 (2009).

[26] T.-L. Ho and Q. Zhou, Squeezing Out the Entropy of Fermions in Optical Lattices, Proc. Natl. Acad. Sci. U.S.A. 106, 6916 (2009). 